\title{
Introducing an Extended Covalent Bond between Oxygen Atoms with an OXO-Shape in Ions and Molecules: Compatibility with the Even-0dd and the Isoelectronicity Rules
}

\author{
Geoffroy Auvert' ${ }^{1}$, Marine Auvert ${ }^{2}$ \\ ${ }^{1}$ Grenoble Alpes University, Grenoble, France \\ ${ }^{2}$ Strasbourg University, Strasbourg, France \\ Email: Geoffroy.auvert@grenoble-inp.org
}

Received 15 June 2016; accepted 13 August 2016; published 16 August 2016

Copyright (C) 2016 by authors and Scientific Research Publishing Inc.

This work is licensed under the Creative Commons Attribution International License (CC BY). http://creativecommons.org/licenses/by/4.0/

(c) (i) Open Access

\begin{abstract}
Building on the recent success of the even-odd rule, the present paper explores its implications by studying the very specific case of OXO compounds. These compounds are usually represented with double bonds linking two oxygen atoms to a central atom-as in carbon dioxyde-yet can sometimes be drawn in a triangular structure, such as in calcium dioxyde. Measurement data moreover indicate that most $0 X 0$ compounds have an angle around $120^{\circ}$ between oxygen atoms, although that seems incompatible with triangular representations. The aim here is to unify these commonly admitted representations by linking oxygen atoms through a single bond that is longer than usual covalent bonds: an "elongated bond". This elongated bond has the interesting effect of suppressing the need for double bonds between oxygen and the central atom. The elongated bond concept is applied to about a hundred of molecules and ions and methodically compared to classical representations. It is shown that this new representation, associated to the even-odd rule, is compatible with all studied compounds and can be used in place of their classical drawings. Its usage greatly simplifies complex concepts like resonance and separated charges in gases. Elongated bonds are also shown to be practicable with the isoelectronic rule as well as isomers, and throughout chemical reactions. This study of an especially long and wide angle bond confirms the versatility of the even-odd rule: it is not limited to compounds with short covalent bonds and can include 00 covalent bond lengths of more than $200 \mathrm{pm}$ and with $0 X 0$ angles above $90^{\circ}$.
\end{abstract}

\section{Keywords}

Elongated Bond, Covalent Bond, Even-Odd, Double Bond, Isoelectronicity, Rules, Chemistry, Ions, Molecule 


\section{Introduction}

In 1789, W. Higgins used a short straight line between two atoms to represent an NO compound [1]. In 1858, A. Scott Cooper used the same line to represent the link between carbon atoms in a molecule [2]. Shortly after, J. Loschmidt extended this representation using more than one line: one line between two carbons in ethane, two lines in ethylene and three lines in acetylene [3]. Between 1900 and 1930, with the development of the periodic table [4] and the discovery of the electron [5], Abegg [6], Lewis [7] and Langmuir [8] proposed a specific rule to represent structural formulas of compounds. The adequately named "octet rule", largely used multi-bonded representations for ions and molecules.

Around 2000 however, Gillespie declared the octet rule to be limited to organic compounds [9] [10]. In 2014, one of the authors of the present paper introduced another representation in order to reconcile representations of organic and inorganic compounds [11]. This representation states that single bonds suffice to draw ions and molecules [11]. This was formalized into a rule, named the "even-odd rule" [12]. Between 2014 and 2016, this rule was theoretically applied to numerous well-known single bonded compounds [12]-[14] and shown to be practicable in all reviewed cases.

The aim of the present paper is to apply the even-odd rule to peculiar ions and molecules that contain oxygen atoms taking an OXO form. In one commonly met representation, the oxygen atoms are linked via double bonds to the central atom. In order to replace all double bonds by single bonds, the authors propose a curved elongated line connecting directly both oxygen atoms.

After a brief reminder of the even-odd rule, the representation of the elongated $\mathrm{OO}$ bond is detailed, and then applied to several molecules with two double bonds. In researching for meaningful molecules, the authors have noticed that several OXO molecules are actually represented in various configurations. This is why the elongated bond is also challenged on these other configurations of OXO triplets. In the ensuing discussion, the potential impacts of this representation are highlighted, including on the concept of resonance.

After a methodical review of a hundred ions and molecules, this paper will finally conclude on the importance of the single-bond representation associated to an elongated covalent bond between atoms for the understanding of OXO chemical structures.

\section{The Even-Odd Rule}

The even-odd rule is associated to single-covalent bonds in ions and molecules [11] and constraints the number of bonds around each atom, depending on its charge and its location in the periodic table. In this rule, atoms are classified into two types: atoms with an even number of electrons are dubbed even-atoms and atoms with an odd number of electrons, odd-atoms. In other words, atoms in columns 2, 4, 6 and 8 of the periodic table are named even-atoms, while those in columns 1, 3, 5 and 7 are odd-atoms.

The even-odd rule was formerly detailed in Ref. [11] and can be summarized as follows.

The rule admits three shells around each atom of a compound: an inner shell, an inactive shell and a covalent shell. In the inner shell, the number of electrons is known from the periodic table and these electrons, organized by pairs, are not involved in any covalent bond. The covalent shell of an atom is composed of electron pairs forming covalent bonds with other atoms. Intercalated between these shells, the inactive shell is composed of electron pairs which can be used, when necessary, to build connections.

Few examples below illustrate representations of atoms using the even-odd rule.

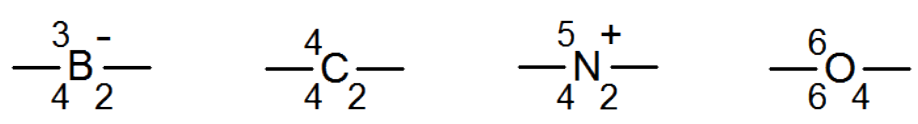

In the above drawings, the center letter originates from the periodic table and each line around an element symbolizes a covalent bond. Each line can thus also be interpreted as a pair of electrons in the covalent shell of the atom. Around the letter, four symbols: the valence number, the charge of the atom, the effective valence number and the number of electrons in the inactive shell. These quantities are evaluated as follows.

The valence number emanates directly from the periodic table and is indicated at the top left of the element name. In our examples, valence numbers are 3, 4, 5 and 6 respectively. At the top right, the sign corresponds to the charge of the atom: a (+) sign means that one electron is missing; a (-) sign that one extra electron is present. 
In the third example, the nitrogen atom has a positive charge and its effective valence number, placed at the bottom left, is equal to 4 . For the boron atom, charged negatively, the effective valence is increased by one and equals to 4. For carbon and oxygen, without charge, i.e. without symbol at the top right, effective valence numbers are equal to the valence numbers of the element. In all cases, the calculated effective valence is indicated at the bottom left of the letter. By subtracting the number of bonds from the effective valence number, the number of electrons in the inactive shell is obtained and shown at the bottom right of the element name. In the examples, the number of electrons in the inactive shell is 4 for oxygen and 2 for the others. As stipulated in the even-odd rule, the number of electrons in the inactive shell is even [11]. This is fully compatible with the concept of electron pair, as first introduced by Lewis in 1916 and repeated in 1923 [15].

In the present paper, when using the even-odd rule for representing compounds, atoms are displayed surrounded by numbers in every corner. In order to avoid confusion and distinguish the number of atoms in a compound from the number of inactive electrons in each atom, chemical formulas will be written $\mathrm{H} 2 \mathrm{O}$ for water or C6H6 for benzene [11]-[14]. In the same way, the total charge of a compound is written in the end: $\mathrm{H} 3 \mathrm{O}(+)$ and $\mathrm{OH}(-)$ for water ions [11]-[14].

\section{Elongated Single Covalent Bond between Two Oxygen Atoms}

A line has historically been used to symbolize a connection between two neighbor atoms in chemical structures.

The present paper postulates that in OXO compounds, oxygen atoms are linked through a longer type of connection, which needs to be represented slightly differently. To symbolize this elongated bond, the authors propose a curved line as illustrated below, which length sets an angle of about $120^{\circ}$ around the $\mathrm{X}$ :
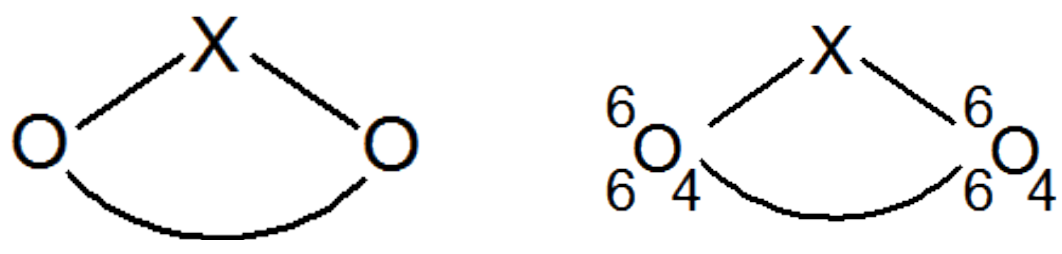

To link both oxygen atoms, a circular arc centered on X represents the elongated covalent bond. Straight lines representing two single covalent bonds connect each oxygen atom with a central atom represented by the letter $\mathrm{X}$. This drawing is conveniently compatible with the theoretical expectation that neutral oxygen atoms should have two covalent bonds.

We will see that this configuration does not prevent the atom $\mathrm{X}$ from being connected to another or several other atoms in addition to the oxygen atoms, in order to constitute compounds commonly met in chemistry.

\section{Comparison with Known Compounds}

In a first attempt to test this new principle, we will look at how this elongated bond affects the representation of known compounds.

For each compound in the following, the drawing on the right is that available in classical references, while the new representation, depicting an elongated bond between atoms of oxygen, is shown on the left. Sources of classical representations are indicated next to the drawing itself. This systematic comparison addresses four different types of commonly found configurations in turn: compounds with two double bonds; compounds with one double bond and one single bond with a linear representation; an association of a double bond with a single bond ending with a charged atom; a triangle with three single bonds. Ions are also addressed in separate chapters.

\subsection{Molecules with Oxygen Atoms Classically Represented with Two Double Bonds}

\subsection{1. $\mathrm{X}$ is an Even-Atom with an Even Number of Bonds}

In Figure 1, all $\mathrm{X}$ atoms are even-atoms, as defined in the periodic table, and have an even number of bonds in agreement with the even-odd rule [11]. In Figure 1, elongated bonds representations are compared to commonly found representations using two double bonds. 


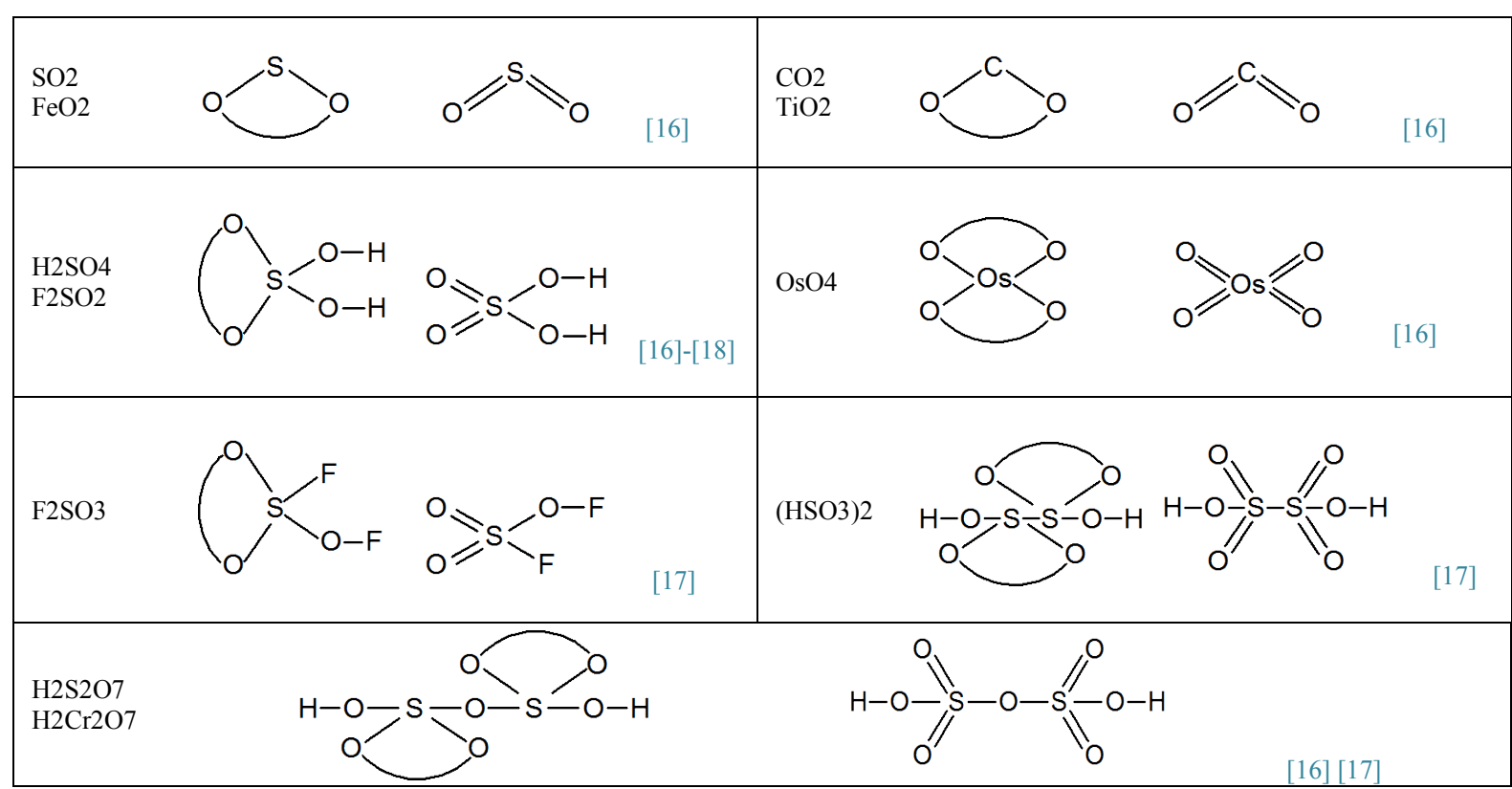

Figure 1. Central even-atoms with an even number of bonds in diverse OXO compounds: comparison of representation with elongated bond (left) and classical representation (right).

Comparing both representations in Figure 1, one can see that atom $\mathrm{X}$ has two fewer bonds when atoms of oxygen are linked via an elongated bond.

\subsection{2. $\mathrm{X}$ is an Odd-Atom with an Odd Number of Bonds}

According to the even-odd rule, an uncharged odd-atom X has an odd number of bonds. Figure 2 compares drawings with elongated bonds to the classical representation using two double bonds.

In some neutral molecules, like for instance $\mathrm{FClO} 3$ or $\mathrm{FBrO} 3$, the elongated bond only connects two of the three oxygen atoms. The third double bond of the classical representation is replaced accordingly to the evenodd rule by a single bond, with a positive and a negative charge at both ends of the line [11].

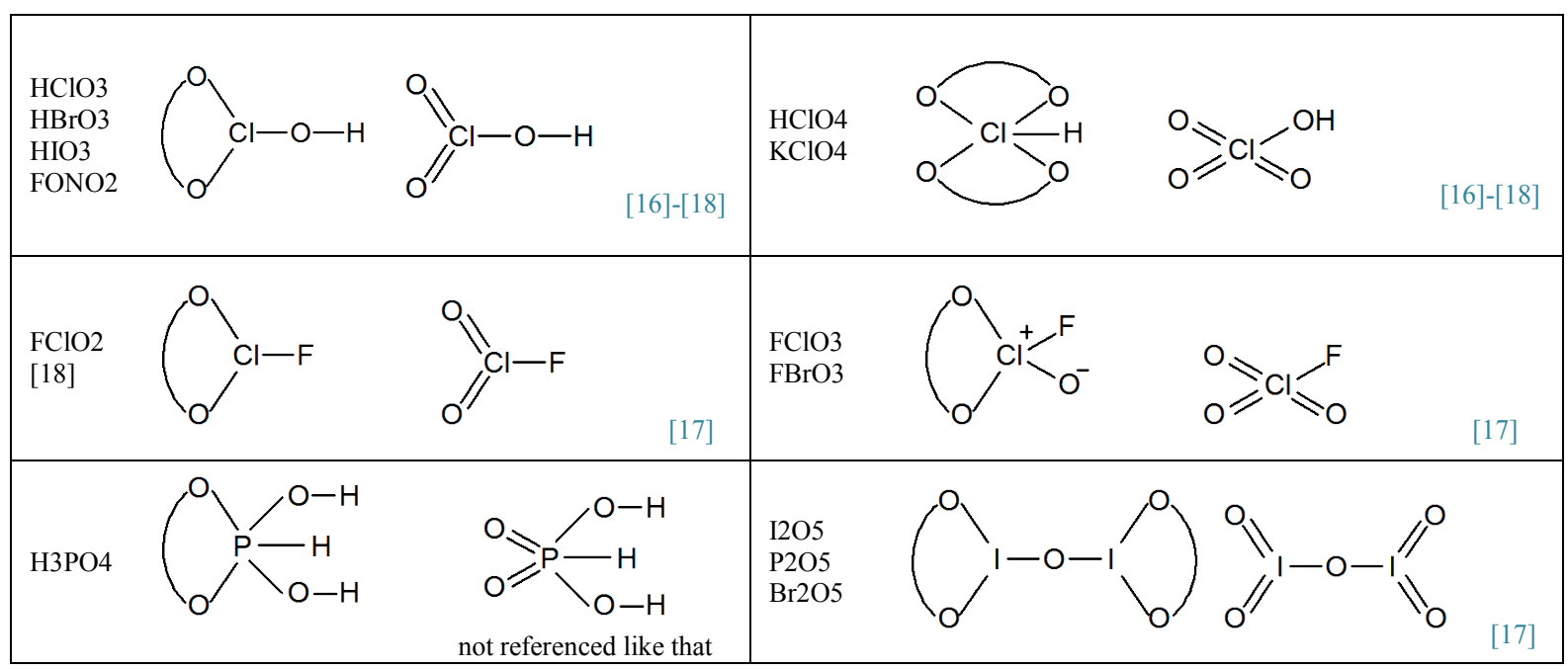

Figure 2. Central odd-atoms with an odd number of bonds in diverse OXO compounds: comparison of representation with elongated bond (left) and classical representation (right).

As in Figure 1, X odd-atoms listed in Figure 2 possess two fewer bonds when atoms of oxygen are con- 
nected through an elongated bond.

\subsection{Molecules Classically Represented by a Linear Model}

Figure 3 compares linear models of molecules with the proposed representation.

$\mathrm{X}$ atoms in Figure 3 are all uncharged odd-atom. According to the even-odd rule, they must possess an odd number of bonds. Compounds illustrated here are classically represented with one atom $(\mathrm{H}$ or $\mathrm{Li})$ connected to an oxygen atom. On the contrary, the even-odd rule relocates these atoms and connects them to the central X atom through a single covalent bond. These proposed OXO geometries are sufficiently different from the classical ones that one could assume experimental data would indicate which configuration reflects the reality.

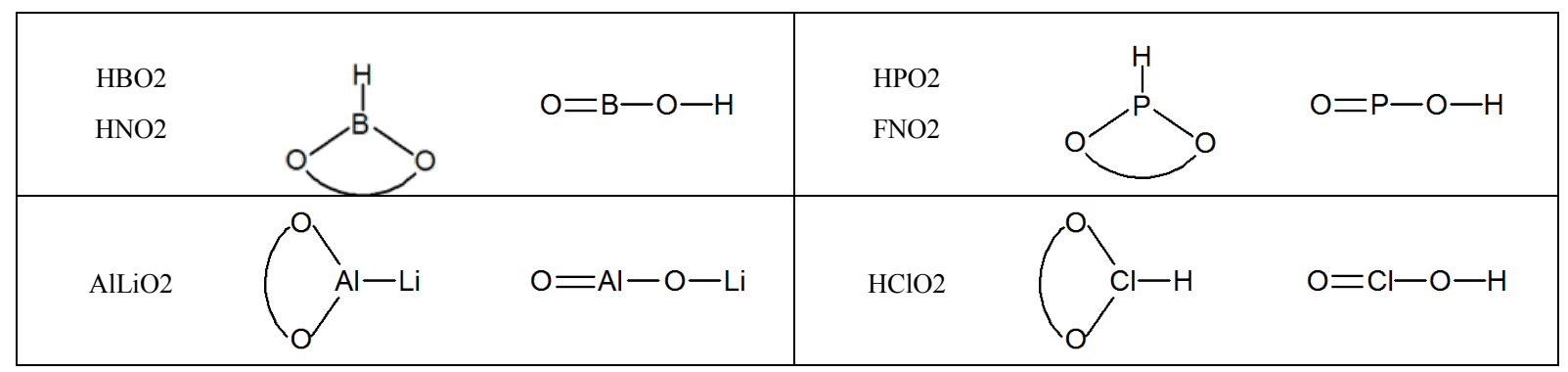

Figure 3. Classical linear model from [16]-[18] modified with elongated OO bonds: comparison of representation with elongated bond (left) and classical representation (right).

\subsection{Molecules Classically Represented with One Double Bond and a Bond Ending with a Charge}

Figure 4 lists molecules with a single bond ending with a charged atom along their representations with an elongated bond. As a reminder on the even-odd rule, an uncharged X odd-atom has an odd number of bonds and an even-atom has an even number of bonds whereas for a charged atom, it is reversed [11].

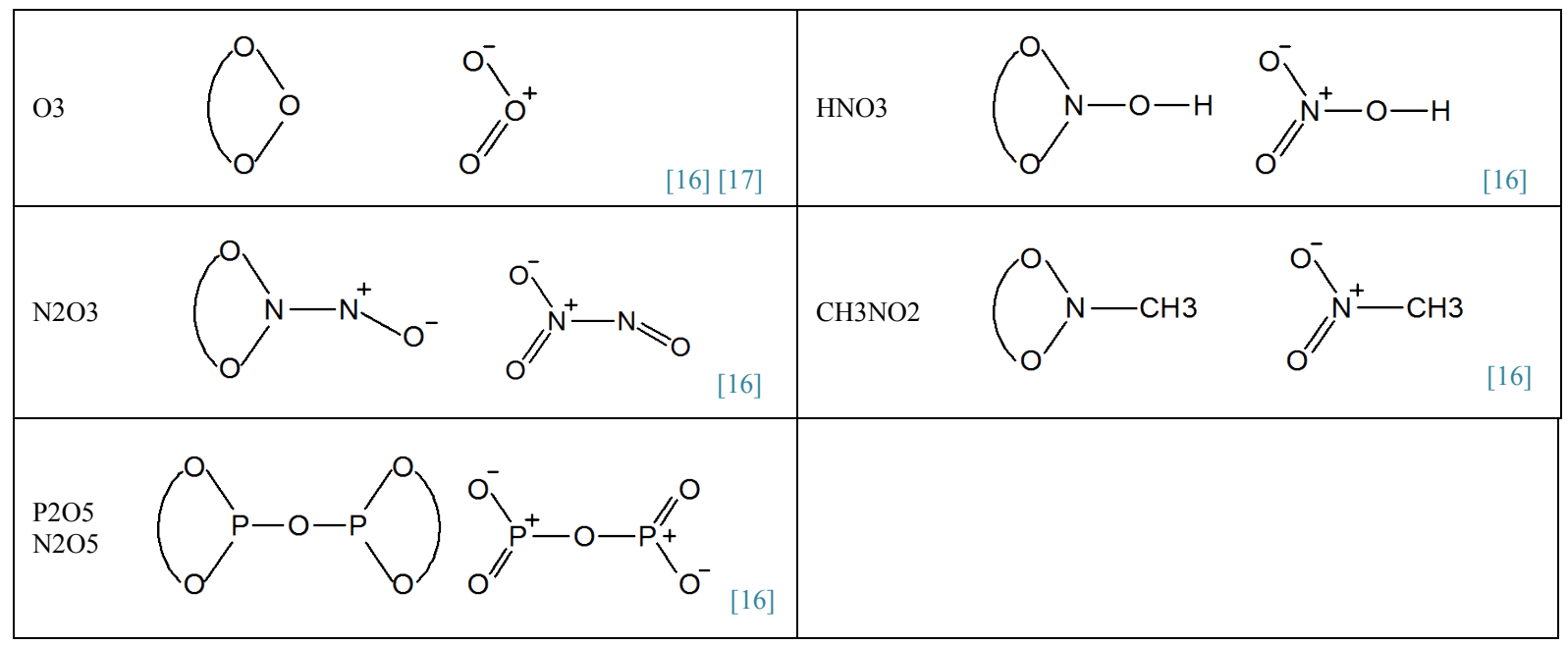

Figure 4. Modifications of classical representations with one double bond and a charged single bond.

In Figure 4, the use of elongated OO bonds does not affect the overall neutrality of the molecule.

\subsection{Molecules Classically Represented with a Triangle}

In Figure 5, classical triangular representations are compared to compounds with an elongated OO bond.

In a triangular representation, convention sets $\mathrm{OXO}$ and $\mathrm{OOX}$ angles around $60^{\circ}$ and an OO length equal to the XO length. 


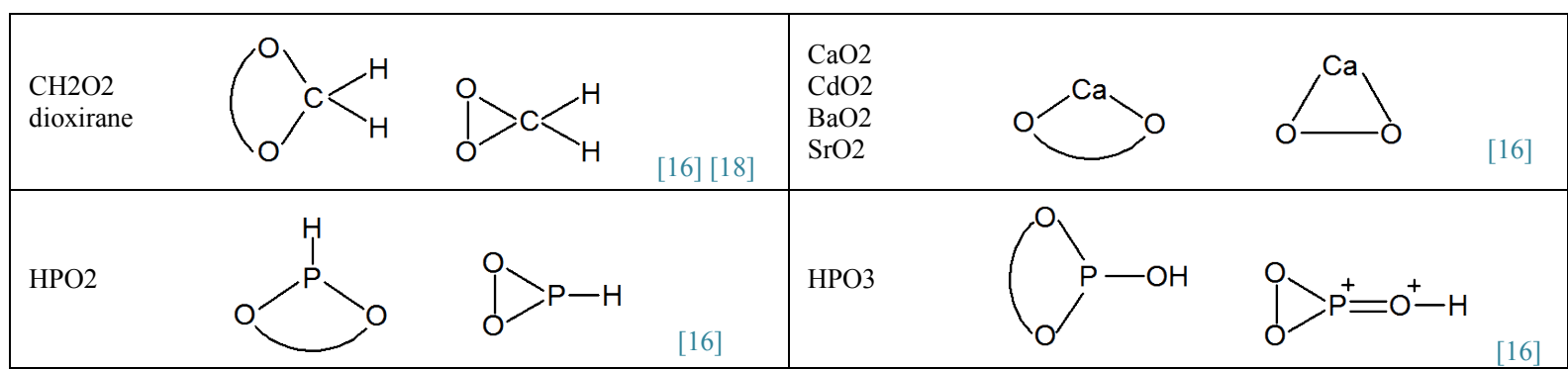

Figure 5. Modifications of classical drawings with a triangle.

On the other hand, elongated bonds define an OXO angle about twice as big and the OO length about twice as long as in the triangle. The discussed values are sufficiently distinctive that experimental data could probably confirm which configuration best reflects reality.

\subsection{Ions Classically Represented with Two Double Bonds}

In this section, representations of the elongated $\mathrm{OO}$ bond in ions are compared with the classical representation for known ions.

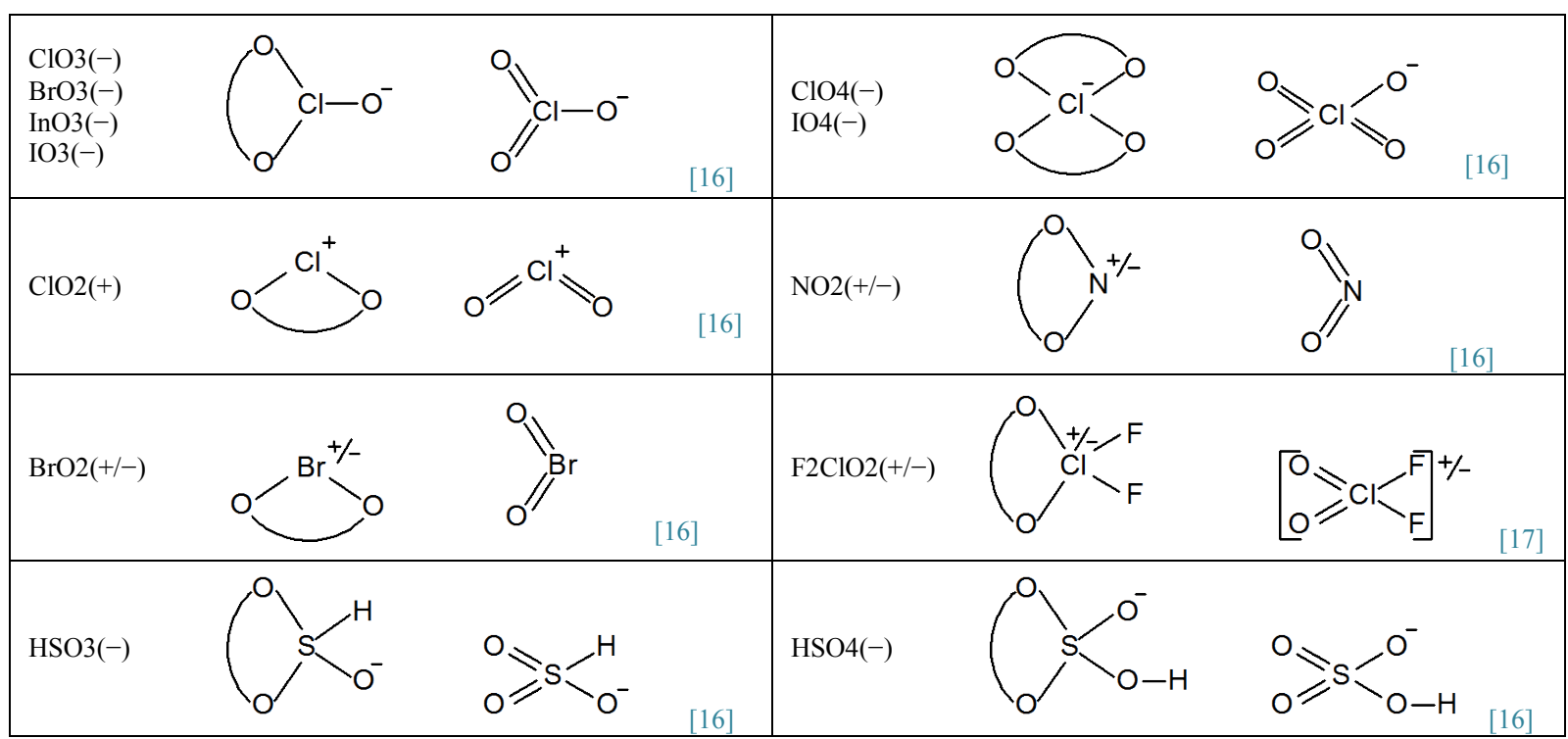

Figure 6. Ions classically represented with two double bonds: comparison of representation with elongated bond (left) and classical representation (right).

In Figure 6, the overall charge of the listed ions is identical across representations. Local charges may however be located at different positions in both models. $\mathrm{ClO} 4(-)$, for instance, remains overall negatively charged, whereas the local charge is once located on an oxygen atom (classical representation), once on the chlorine atom (representation with elongated bond).

\subsection{Ions Classically Represented with a Double Bond and a Single Bond Ending with a Charge}

A few known charged compounds are depicted in Figure 7, on the left with elongated bonds and on the right in their classical double-bond representation.

It is worth noting that the symmetrical character of the elongated bond establishes a global symmetry for the ions in Figure 7. The single bond ending with a charged oxygen atom does not allow this characteristic. 


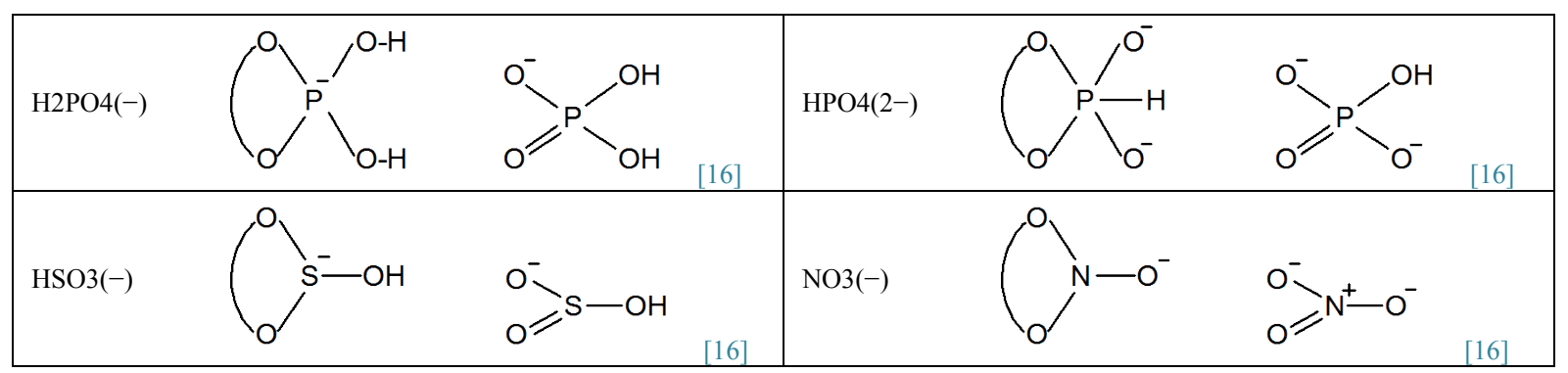

Figure 7. Ions classically represented with one double bond and a single bond ending with a charge: comparison of representation with elongated bond (left) and classical representation (right).

As in chapter 4.5, local charge positions may not be the same in both models but the total charge is maintained across representations.

In the review carried out in paragraphs 4.1 to 4.6 , all classical double bonds were smoothly replaced by single bonds, in agreement with the even-odd rule [11]. This systematic review of 60 molecules and ions leads the authors to suggest that the proposed representation with elongated $\mathrm{OO}$ bonds unifies the commonly found representations of the structure of OXO ions and molecules.

\section{Discussion}

In the following, we discuss the repercussions of a representation with elongated bonds on different familiar topics of chemistry, such as bond lengths, resonance, isomers, multi-charges, isoelectronic and chemical reactions.

\subsection{Bond Length in OXO Form}

An ozone molecule, illustrated in Figure 4, can be a reference for the geometry of an OXO compound. Microwave measurements on this molecule indicate an angle of about $120^{\circ}$ for the OXO angle and an OX bond length of about $130 \mathrm{pm}$ [17]. Measures on the SO2 molecule give nearly the same values [17]. Geometrically, we can deduce that oxygen atoms are about $220 \mathrm{pm}$ apart.

Since the hypothesis of the present article is that the elongated bond is a circular arc binding both oxygen atoms and whose center is the $\mathrm{X}$ atom, the length of the curved line is around $260 \mathrm{pm}$. For the same reason, the $\mathrm{XOO}$ angle is slightly above $90^{\circ}$ in ions and molecules.
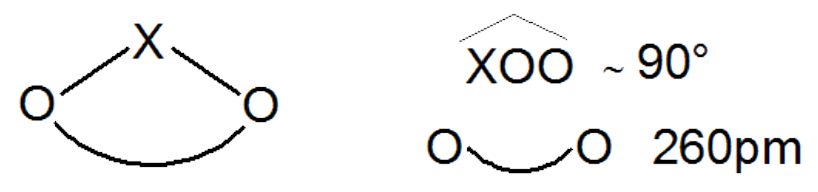

\subsection{Resonance Structure or Mesomerism in Chemistry}

Resonance has been described as a temporary superposition of a double bond and a single bond in a compound [19]. Resonance is a concept that has actually been proposed to accommodate valid experimental results to classical multi-bonded drawings of compounds [20]. In CO3 for instance and as shown below, the three oxygen atoms do not have the same bonding conditions in the three classical representations. One bond is double while the other two are single. The concept of resonance is an average of every possible drawing (depicted on the right).

$\mathrm{CO} 3(2-)$

$\mathrm{SO} 3(2-)$

[18]

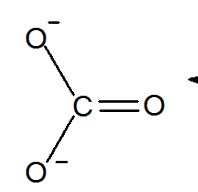<smiles>C[C-]C(=O)O[C-]C(=O)[O-]</smiles>

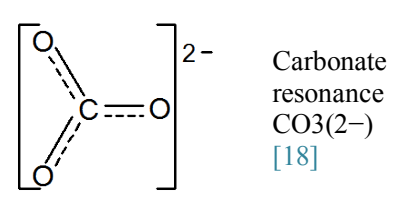




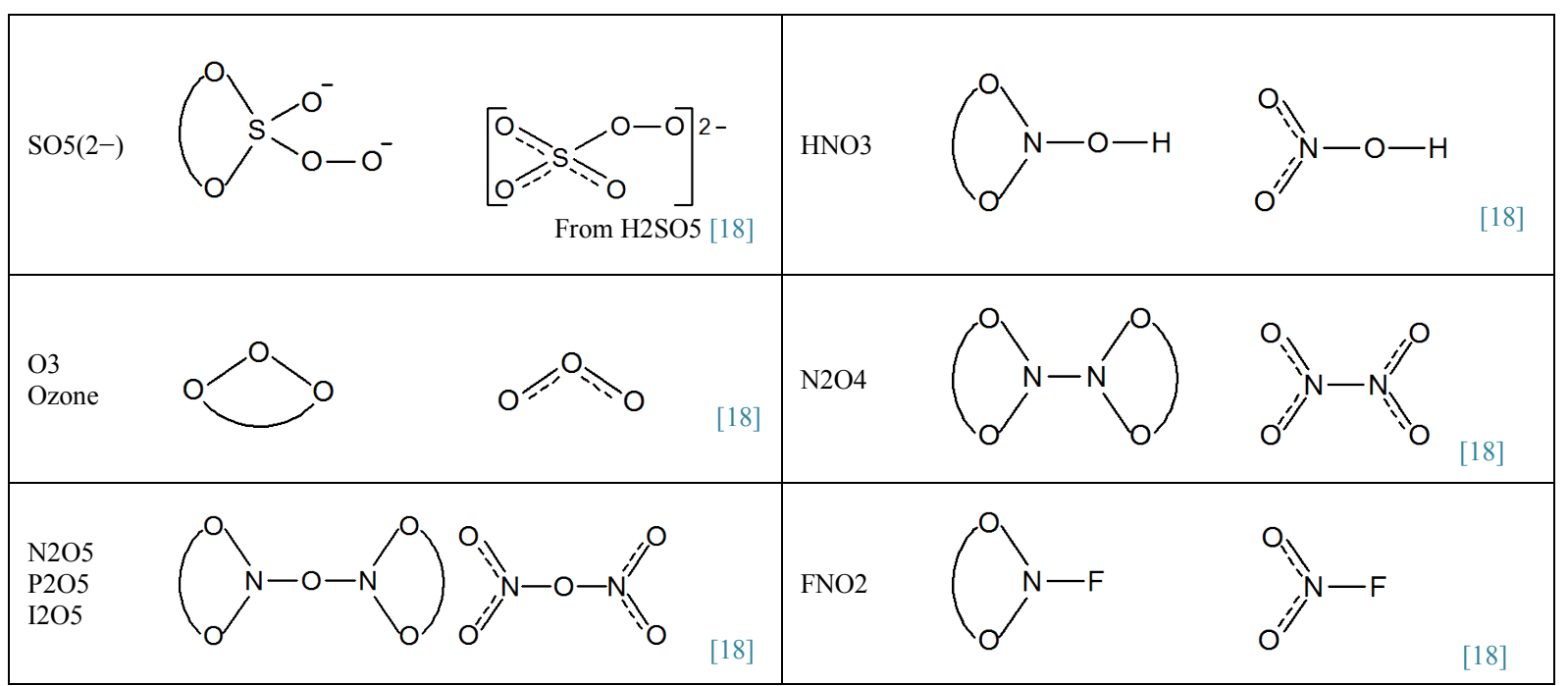

Figure 8. Classical resonance drawing with two lines; a dashed line along a continuous line (right), compared to the drawing of an elongated $\mathrm{OO}$ bond without resonance (left).

In Figure 8, we compare classical drawings of gaseous compounds, usually described using resonance, with the proposed representation including an elongated $\mathrm{OO}$ bond (left side).

The review carried out on molecules of Figure 8 indicates that the concept of resonance might be irrelevant when using an elongated $\mathrm{OO}$ bond.

Interestingly, a similar conclusion was drawn in a previous publication, in which the even-rule was extended to suppress double bonds in a selected number of ions and molecules [11]. For the studied ions and molecules, resonance was considered superfluous as well.

Applied to an ion like $\mathrm{CO} 3(2-)$ for instance, the ion used at the beginning of this chapter to illustrate the concept of resonance, the even-odd rule greatly simplifies the representation as:

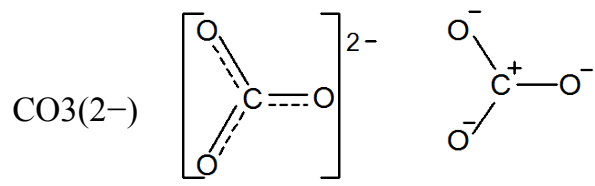

These different examples lead the authors to suggest that the single bond constraint imposed by the even-odd rule would render both concepts of multi-bonding and mesomerism altogether unnecessary.

\subsection{Isomers}

Two compounds are said to be isomeric when they are composed of the same atoms located at different positions or have different bonding arrangements [21]. Figure 9 exhibits two isomeric compounds having the same name and a different arrangement.

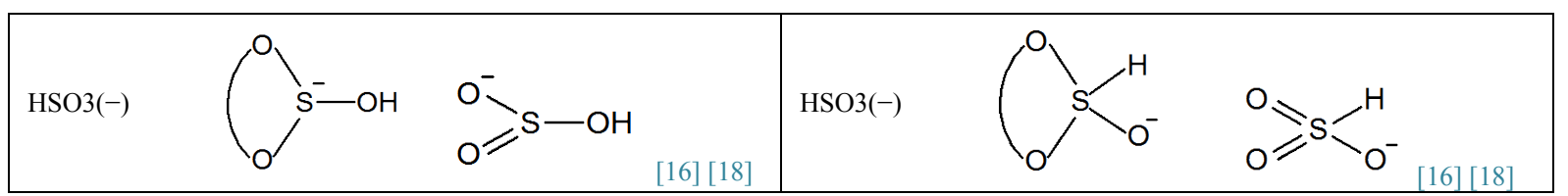

Figure 9. Comparison of representations using an elongated $\mathrm{OO}$ bond (left) compared with their classical drawings (right) for two isomers of $\mathrm{HSO} 3(-)$.

Strikingly, in Figure 9 like in all examples above, the elongated OO bond geometry is not affected by a change of the other atoms of the compounds. 


\subsection{Neutral Compounds Classically Represented with Multi-Charged Atoms}

Some compounds are commonly represented with multi-charged atoms. By contrast, the even-odd rule agrees with single charges affected to any atoms in a compound but not with any atoms bearing multiple charges. Figure 10 illustrates how the use of the elongated covalent bond together with the even-odd rule suppresses the need for multiple charges.

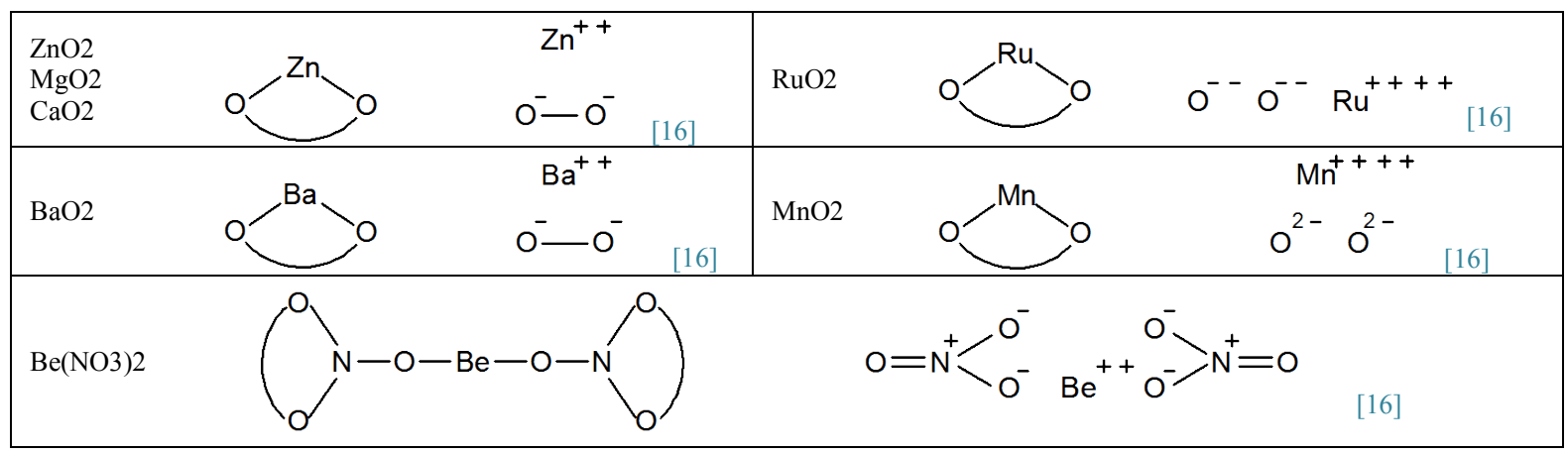

Figure 10. Comparison of classical multi-charged representations with elongated $\mathrm{OO}$ bonds in neutral compounds.

In Figure 10, classical representations of gases seem to lack bonding and let charged atoms free to float around. The use of an elongated bond provides an elegant representation for these specific neutral compounds.

This list also contains interesting cases of molecules that can also be found in various configurations. $\mathrm{CaO} 2$ for instance was already listed as a triangular shape in Figure 5. $\mathrm{MnO} 2$ can be found represented with double bonds similar to those of Figure 1. The elongated bond representation could replace these numerous configurations with a unique representation.

\subsection{Isoelectronicity}

An isoelectronicity rule was previously proposed in association with the even-odd rule [22]. This rule details how to replace an atom by another without affecting its bonding configuration. In Figure 11, the isoelectronicity rule is applied to both proposed and classical structures and result compared. Compounds on the same row are isoelectronic.

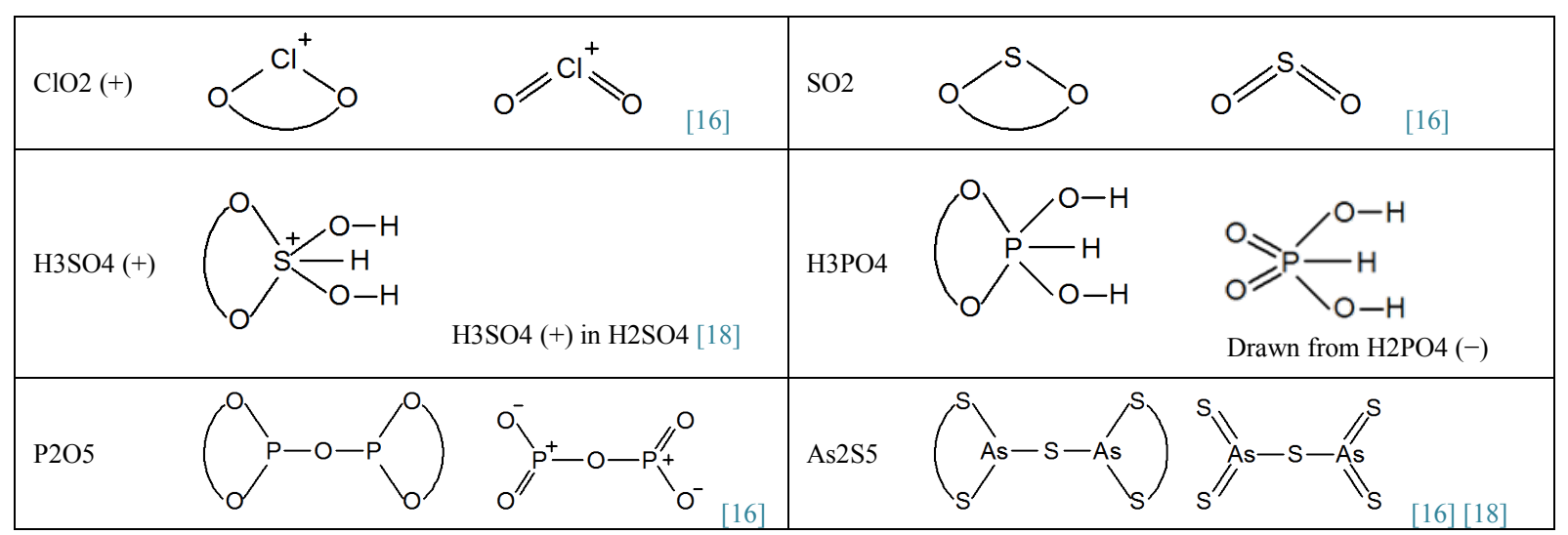

Figure 11. Use of elongated bond on isoelectronic compounds. Compounds on the same row are isoelectronic: $\mathrm{Cl}(+) / \mathrm{S}, \mathrm{S}(+)$ $/ \mathrm{P}$ and $\mathrm{P} / \mathrm{As}$ are pairs of isoelectronic atoms.

In Figure 11, $\mathrm{P} 2 \mathrm{O} 5$ and As2S5 are isoelectronic whereby elements $\mathrm{O}$ and $\mathrm{P}$ are respectively isoelectronic with $\mathrm{S}$ and As, each pair belonging to the same column of the periodic table. Other compounds agree with the isoelectronicity rule detailed in Ref. [22].

It is worth noting that elongated bonds configuration is compatible with isoelectronicity. 


\subsection{Chemical Reactions}

Considering two reactions described in [17], phosphoric acid with water-page 518 or sulfuric acid with waterpage 711, Figure 12 and Figure 13 depict the evolution of the compound representation with elongated bond throughout both groups of reactions.

\begin{tabular}{|l}
\hline \\
\hline \\
$\mathrm{H} 2 \mathrm{SO} 4+\mathrm{H} 2 \mathrm{O} \longrightarrow \mathrm{HSO} 4(-)+\mathrm{H}_{3} \mathrm{O}(+)$ \\
$\mathrm{HSO}(-)+\mathrm{H}_{2} \mathrm{O} \longrightarrow \mathrm{SO} 4(2-)+\mathrm{H} 3 \mathrm{O}(+)$ \\
$\mathrm{SO} 4(2-)$
\end{tabular}

Figure 12. The elongated $\mathrm{OO}$ bond remains unaffected throughout the chemical reactions between sulfuric acid and water.

Throughout both groups of reactions, it becomes clear that the structure of OO elongated bonds is not influenced by the modification of other ends of the compounds.

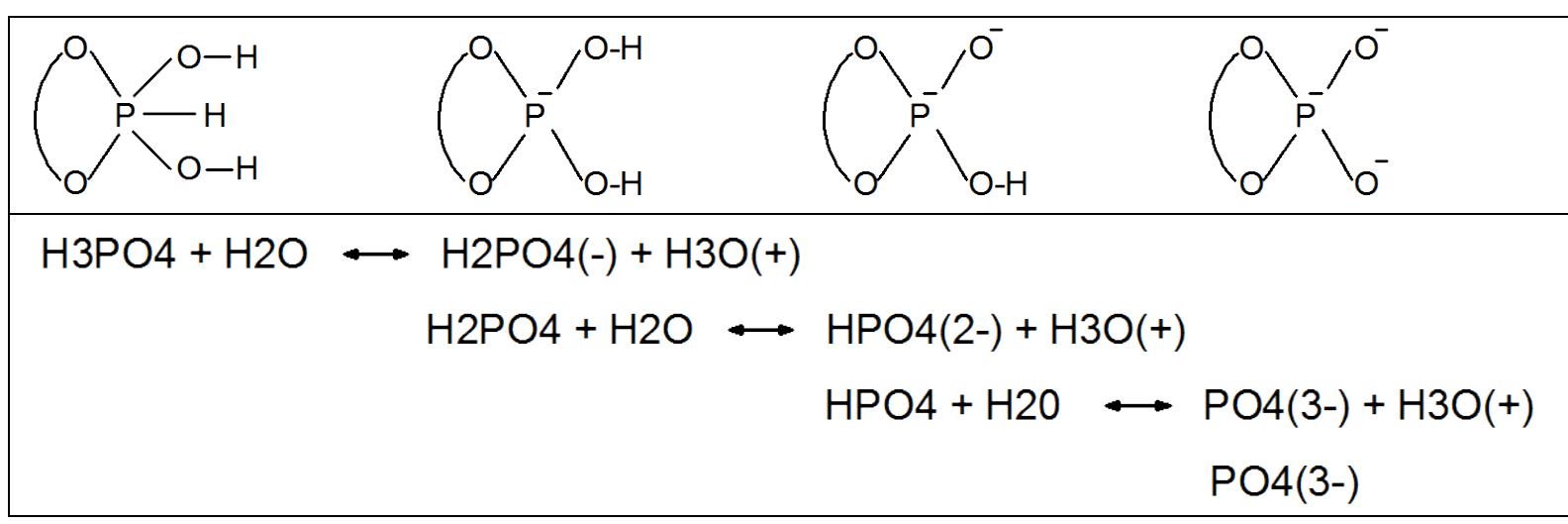

Figure 13. The elongated $\mathrm{OO}$ bond remains unaffected throughout the chemical reactions between phosphoric acid and water.

In an attempt to summarize the findings from these discussions, it seems that the proposed representation addresses several important topics in chemistry: it explains experimental measurements on OXO compounds and behavior in chemical reactions, suppresses complex expedients like resonance structures and molecules classically represented with separated charges and finally, agrees with isomers and isoelectronic compounds.

The authors conclude therefore that the even-odd rule applies to short or extended single-covalent bonds in structural formulas of numerous ions and molecules.

\section{Conclusion}

The most common representation of a covalent bond is a line placed between two atoms that are in effect about $100 \mathrm{pm}$ apart. In an OXO form, the distance between the oxygen atoms is above $200 \mathrm{pm}$ and conventional representations only propose that both oxygen atoms are bonded with the $\mathrm{X}$ atom. Building on the recent success of the even-odd rule, the authors suggest that several classical representations of OXO forms could be modified using a curved line linking both oxygen atoms, which could be named "elongated bond". It was shown that this new representation, associated to the even-odd rule, was compatible with numerous compounds and could be used in place of their classical drawings. Its usage was compared to that of resonance and separated charges in gases and displayed in both cases more elegant solution. In the same way, the proposed representation was shown to be compatible with the associated isoelectronic rule and with isomers. The authors conclude that the 
elongated bond is applicable to many compounds and remains practicable throughout chemical reactions. This study of an especially long and wide angle bond confirms the versatility of the even-odd rule: it is not limited to compounds with short covalent bonds and can include OO covalent bond lengths of more than $200 \mathrm{pm}$ and with OXO angles above $90^{\circ}$.

\section{References}

[1] Higgins, W. (1814) Observations and Experiments on the Atomic Theory and Electrical Phenomena. Complete Dictionary of Scientific Biography, 20 June 2015. http://www.encyclopedia.com/doc/1G2-2830901997.html

[2] Couper, A.S. (1858) Sur une nouvelle théorie chimique. Annales de Chimie et de Physique, 53, 469-489.

[3] Bader, A. and Parker, L. (2001) Biography of Joseph Loschmidt. Physics Today, March 2001. http://www.loschmidt.cz/loadframe.html?biography.html

[4] Mendeleev, D. (1901) The Principles of Chemistry. Collier, New York.

[5] Thomson, J.J. (1897) Cathode Rays. Philosophical Magazine, 44, 293-316. http://dx.doi.org/10.1080/14786449708621070

[6] Abegg, R. (1904) Die Valenz und das periodische System. Zeitschrift für Anorganische Chemie, 39, 330-380. http://dx.doi.org/10.1002/zaac.19040390125

[7] Lewis, G.N. (1916) The Atom and the Molecule. Journal of the American Chemical Society, 38, 762-785. http://dx.doi.org/10.1021/ja02261a002

[8] Langmuir, I. (1919) The Arrangement of Electrons in Atoms and Molecules. Journal of the American Chemical Society, 41, 868-934. http://dx.doi.org/10.1021/ja02227a002

[9] Gillespie, R.J. (2004) Teaching Molecular Geometry with the VSEPR Model. Journal of Chemical Education, 81, 298304. http://dx.doi.org/10.1021/ed081p298

[10] Gillespie, R.J. and Popelier, P.L.A. (2001) Chemical Bonding and Molecular Geometry: From Lewis to Electron Densities. Oxford University Press, Oxford.

[11] Auvert, G. (2014) The Even-Odd Rule on Single Covalent-Bonded Structural Formulas as a Modification of Classical Structural Formulas of Multiple-Bonded Ions and Molecules. Open Journal of Physical Chemistry, 4, 173-184. http://dx.doi.org/10.4236/ojpc.2014.44020

[12] Auvert, G. (2015) How the Even-Odd Rule, by Defining Electrons Pairs and Charge Positions, Can Be Used as a Substitute to the Langmuir-Octet Rule in Understanding Interconnections between Atoms in Ions and Molecules. Open Journal of Physical Chemistry, 5, 28-38. http://dx.doi.org/10.4236/ojpc.2015.52004

[13] Auvert, G. (2014) Improvement of the Lewis-Abegg-Octet Rule Using an "Even-Odd" Rule in Chemical Structural Formulas: Application to Hypo and Hyper-Valences of Stable Uncharged Gaseous Single-Bonded Molecules with Main Group Elements. Open Journal of Physical Chemistry, 4, 60-66. http://dx.doi.org/10.4236/ojpc.2014.42009

[14] Auvert, G. (2014) Chemical Structural Formulas of Single-Bonded Ions Using the "Even-Odd" Rule Encompassing Lewis's Octet Rule: Application to Position of Single-Charge and Electron-Pairs in Hypo- and Hyper-Valent Ions with Main Group Elements. Open Journal of Physical Chemistry, 4, 67-72. http://dx.doi.org/10.4236/ojpc.2014.42010

[15] Lewis, G.N. (1923) Valence and the Structure of Atoms and Molecules. American Chemical Monograph Series, the Chemical Catalog Co., Inc., New York.

[16] http://www.chemspider.com/

[17] Greenwood, N.N. and Earnshaw, A. (1998) Chemistry of the Elements. 2nd Edition, Butterworth-Heinemann, Oxford.

[18] https://en.wikipedia.org/wiki/English Wikipedia

[19] Pauling, L. (1960) The Nature of the Chemical Bond - An Introduction to Modern Structural Chemistry. 3rd Edition, Cornell University Press, Ithaca, New York, 10-13.

[20] http://en.wikipedia.org/wiki/Resonance (chemistry)

[21] Crum Brown, A. (1865) On the Theory of Isomeric Compounds. Journal of the Chemical Society, 18, 230-245. http://dx.doi.org/10.1039/JS8651800230

[22] Auvert, G. (2014) Coherence of the Even-Odd Rule with an Effective-Valence Isoelectronicity Rule for Chemical Structural Formulas: Application to Known and Unknown Single-Covalent-Bonded Compounds. Open Journal of Physical Chemistry, 4, 126-133. http://dx.doi.org/10.4236/ojpc.2014.43015 


\section{Submit or recommend next manuscript to SCIRP and we will provide best service for you:}

Accepting pre-submission inquiries through Email, Facebook, LinkedIn, Twitter, etc.

A wide selection of journals (inclusive of 9 subjects, more than 200 journals)

Providing 24-hour high-quality service

User-friendly online submission system

Fair and swift peer-review system

Efficient typesetting and proofreading procedure

Display of the result of downloads and visits, as well as the number of cited articles

Maximum dissemination of your research work

Submit your manuscript at: http://papersubmission.scirp.org/ 